\title{
Clinical outcomes in patients with Philadelphia chromosome-positive leukemia treated with ponatinib in routine clinical practice-data from a Belgian registry
}

\author{
Timothy Devos ${ }^{1}$ (D) V Violaine Havelange ${ }^{2} \cdot$ Koen Theunissen $^{3} \cdot$ Stef Meers $^{4} \cdot$ Fleur Samantha Benghiat $^{5}$. \\ Alain Gadisseur $^{6} \cdot$ Gaëtan Vanstraelen $^{7} \cdot$ Hélène Vellemans ${ }^{8} \cdot$ Benjamin Bailly $^{9} \cdot$ Nikki Granacher $^{10}$. \\ Philippe Lewalle ${ }^{11} \cdot$ Ann De Becker ${ }^{12} \cdot$ Koen Van Eygen $^{13} \cdot$ Mia Janssen ${ }^{14} \cdot$ Agnes Triffet $^{15} \cdot$ Inge Vrelust $^{16}$. \\ Dries Deeren ${ }^{17} \cdot$ Dominiek Mazure $^{18}$. Julie Bekaert ${ }^{19} \cdot$ Michael Beck $^{20} \cdot$ Dominik Selleslag $^{21}$
}

Received: 28 July 2020 / Accepted: 3 April 2021 / Published online: 4 May 2021

(C) The Author(s) 2021

\begin{abstract}
Data on clinical use of ponatinib are limited. This prospective registry aimed to evaluate outcomes of ponatinib treatment in routine practice over 3 years (2016-2019) in Belgium (NCT03678454). Patients with chronic myeloid leukemia (CML) or Philadelphia chromosome-positive $(\mathrm{Ph}+)$ acute lymphoblastic leukemia (ALL) were treated with ponatinib per current label. Fifty patients (33 CML and $17 \mathrm{Ph}+\mathrm{ALL}$ ) were enrolled. Fifty-five percent of CML and 29\% of Ph+ ALL patients had received $\geq 3$ prior tyrosine kinase inhibitors (TKIs). Reasons for starting ponatinib were intolerance (40\%), relapse or refractoriness (28\%) to previous TKIs, progression (16\%), or T315I mutation (16\%). Median follow-up was 15 months for $\mathrm{CML}$ and 4.5 months for Ph+ ALL patients. Best response was a major molecular response in $58 \%$ of CML and $41 \% \mathrm{of} \mathrm{Ph}+$ ALL patients. Of 20 patients who started ponatinib due to intolerance to previous TKIs, 9 (64\%) CML and 4 (67\%) $\mathrm{Ph}+$ ALL achieved a major molecular response. Three-year estimates of overall survival were $85.3 \%$ and $85.6 \%$, respectively, in $\mathrm{CML}$ and $\mathrm{Ph}+\mathrm{ALL}$ patients; estimated progression-free survival was $81.6 \%$ and $48.9 \%$. Adverse reactions were reported in 34 patients (68\%); rash (26\%) and dry skin (10\%) were most common. Reported cardiovascular adverse reactions included vascular stenosis (3), arterial hypertension (2), chest pain (1), palpitations (1), and vascular occlusion (1). This Belgian registry confirms results from the PACE clinical trial and supports routine ponatinib use in CML and Ph+ ALL patients who are resistant or intolerant to previous TKIs or with the T315I mutation.
\end{abstract}

Timothy Devos

timothy.devos@uzleuven.be

1 Department of Hematology, University Hospitals Leuven and Department of Microbiology and Immunology, Laboratory of Molecular Immunology (Rega Institute), KU Leuven, Campus Gasthuisberg, Herestraat 49, B-3000 Leuven, Belgium

2 UCL Saint-Luc, Woluwe-Saint-Lambert, Belgium

3 Jessa Ziekenhuis, Hasselt, Belgium

4 Algemeen Ziekenhuis Klina, Brasschaat, Belgium

5 Hôpital Erasme, Bruxelles, Belgium

6 Universitair Ziekenhuis Antwerpen, Edegem, Belgium

7 CHR Verviers, Verviers, Belgium

8 CHU UCL Namur, Site Godinne, Yvoir, Belgium

9 Hôpital de Jolimont, Haine-Saint-Paul, Belgium Bruxelles, Belgium

12 Universitair Ziekenhuis Brussel, Jette, Belgium

13 Algemeen Ziekenhuis Groeninge, Kortrijk, Belgium

14 Ziekenhuis Oost-Limburg, Genk, Belgium

15 Centre Hospitalier Universitaire Charleroi Vésale, Charleroi, Belgium

16 Algemeen Ziekenhuis Sint-Elisabeth, Turnhout, Belgium

17 Algemeen Ziekenhuis Delta, Roeselare, Belgium

18 Universitair Ziekenhuis Gent, Gent, Belgium

19 Incyte Biosciences International sàrl, Morges, Switzerland

20 Incyte Biosciences Benelux B.V., Amsterdam, The Netherlands

21 Algemeen Ziekenhuis Sint-Jan Brugge, Brugge, Belgium 
Keywords Ponatinib $\cdot$ Routine clinical practice $\cdot$ Registry $\cdot$ Chronic myeloid leukemia $\cdot$ Philadelphia chromosome-positive acute lymphoblastic leukemia

\section{Introduction}

Treatment with tyrosine kinase inhibitors (TKIs) has led to a major improvement in the prognosis of patients with chronic myeloid leukemia (CML) and Philadelphia chromosomepositive $(\mathrm{Ph}+)$ acute lymphoblastic leukemia (ALL) [1]. TKIs selectively target the constitutively active BCR-ABL1 tyrosine kinase, therefore suppressing the growth of malignant cells [2]. One of the challenges in TKI therapy is the development of resistance, in many cases through the presence or acquisition of mutations in the ABL kinase domain [2]. In addition, some patients become intolerant to TKI treatment by developing adverse events (AEs) that cannot always be managed through dose reductions or symptomatic treatment [3]. These AEs are a real challenge in the daily practice of hematologists.

Currently, several TKIs are used in clinical practice, i.e., imatinib, nilotinib, dasatinib, bosutinib, and ponatinib [4]. Ponatinib is a third-generation TKI with potent activity against native and mutated BCR-ABL1 containing single point mutations, including the T315I mutation, for which no other marketed TKI is effective $[5,6]$. Ponatinib received marketing approval based on the results of the phase 2 Ponatinib $\mathrm{Ph}+$ ALL and CML Evaluation (PACE) trial, which demonstrated the efficacy and safety of ponatinib in patients with CML or $\mathrm{Ph}+\mathrm{ALL}$ who were intolerant or resistant to previous lines of TKI therapy [7, 8]. By 12 months, 56\% of chronic phase (CP)CML patients achieved a major cytogenetic response (MCyR; primary endpoint) [7]. The 5-year follow-up data of this trial showed deep and durable responses to ponatinib therapy in $\mathrm{CP}$ CML patients [8]. In total, $60 \%$ of the evaluable CP-CML patients achieved MCyR, and the probability of maintaining MCyR for 5 years was $82 \%$. Fifty-four percent of evaluable CP-CML patients achieved a complete cytogenetic response (CCyR) and $40 \%$ achieved a major molecular response (MMR, defined as BCR-ABL1 mRNA levels $\leq 0.1 \%$ on the International Scale). Ponatinib dose reductions were proactively implemented during the course of the trial to decrease the risk of arterial occlusive events (AOEs). The response to therapy was maintained 40 months after ponatinib dose reduction by $\geq 90 \%$ of patients who had achieved MCyR or MMR. Fiveyear estimated rates of progression-free survival (PFS) and overall survival (OS) were $53 \%$ and $73 \%$, respectively [8]. In $\mathrm{Ph}+\mathrm{ALL}$ patients, the efficacy data demonstrated promising results, with $41 \%$ of patients having a major hematologic response, 47\% MCyR, and 38\% CCyR [8].

The most common treatment-emergent AEs reported in the PACE trial were rash, abdominal pain, thrombocytopenia, headache, dry skin, and constipation. AOEs received special attention in the clinical trial, as they can provoke dramatic consequences for the patients. The cumulative incidence of AOEs in all patients was $17.1 \%(11.8 \%$ serious $\mathrm{AOE})$ in the initial report and $25 \%$ (20\% serious AOE) in the 5-year follow-up of the PACE trial. The risk for AOE incidence appeared to be related to ponatinib dose and pre-existing cardiovascular risk factors $[7,8]$.

Despite ponatinib's authorization in Europe since 2013 [9], data on the use of ponatinib in routine clinical practice are scarce. Nevertheless, collection of such data is important to evaluate the effectiveness and safety of ponatinib and may help optimize the use of ponatinib in daily practice. This registry is, to our knowledge, the first report of prospective data of patients with CML or Ph+ ALL treated with ponatinib in routine clinical practice.

\section{Methods}

\section{Registry design and patients}

This multi-center, prospective, observational study is currently ongoing at 20 centers throughout Belgium (clinicaltrials.gov identifier: NCT03678454). Patients $\geq 18$ years old, diagnosed with CML (all phases) or Ph+ ALL, and who started ponatinib treatment according to the indication in the product label [9] between March 1, 2016, the date of reimbursement in Belgium, and March 1, 2019, were eligible for this enrolment period. In addition, patients, who had participated in a named patient program (NPP) for ponatinib prior to reimbursement but were still being treated with ponatinib on March 1, 2016, were also allowed to participate. Patients were excluded if they participated in a clinical trial at any time during the registry period (Fig. 1).

This registry was conducted in accordance with the Declaration of Helsinki and the International Council for Harmonization guidelines for good clinical practice. All patients provided written informed consent before data collection, but a waiver was granted by the ethics committee (EC) for NPP patients who were still on ponatinib treatment on March 1, 2016, but deceased before the registry start. The registry protocol and the informed consent form were approved by the central EC and local ECs at each participating center; any subsequent amendments were approved by the central EC and notified to local ECs. 


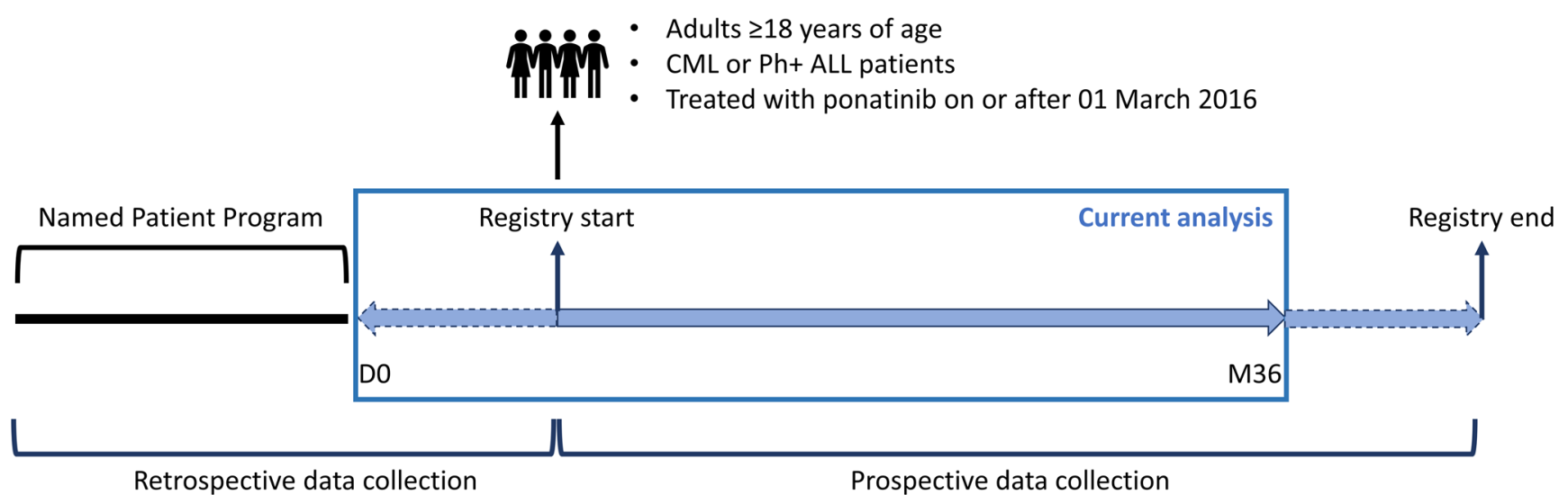

Fig. 1 Study design. D, day; M, month; CML, chronic myeloid leukemia; Ph+ ALL, Philadelphia chromosome-positive acute lymphoblastic leukemia; D0, the start of reimbursement of ponatinib in Belgium, i.e., March 1, 2016

All procedures related to registry conduct, including data management, statistical analysis, and scientific writing, were performed by Modis, Wavre, Belgium.

\section{Data collection}

All data used in the current analysis were collected as of treatment initiation. Data from registry start (which occurred approximately 10 months after reimbursement date) until month 36 were collected prospectively. As treatment initiation could be earlier than registry start, data between March 1, 2016 (date of reimbursement) and registry start were collected retrospectively. For the 8 patients treated in the NPP and still on treatment at registry start, data that had been collected in the NPP were transferred to the database for this registry. Safety data were not collected during the NPP. However, for NPP patients who participated in the registry, safety data were also collected retrospectively between March 1 , 2016 and registry start and prospectively as of registry start.

Data were collected during routine treatment visits in the hospital or at least every 6 months. Documentation and date of informed consent, participation in NPP, patient demographics, disease characteristics, medical history, treatment patterns, treatment outcomes, and safety were collected. All AEs had to be reported based on a pre-defined AE list (Supplementary Table S1), with the option to describe any AE not included in the list, and were followed up until the outcome of the event was established. For all AEs, a possible causal relationship to ponatinib was assessed by the investigator. Responses to treatment for each patient were measured as per routine clinical practice and were defined according to the European LeukemiaNet 2013 recommendations [10]. Mutation testing was done as per routine clinical practice [10].

\section{Data analysis}

Data were analyzed using descriptive statistics. OS and PFS curves were generated using the Kaplan-Meier method. The sample size was not pre-defined. The primary analysis was performed on the overall population. To identify any potential selection bias due to inclusion of patients who had participated to the NPP, a secondary analysis was performed excluding these patients. Subgroup analyses were also performed by disease (Ph+ ALL versus CML), and within the disease for those who started ponatinib due to intolerance or another reason than intolerance (i.e., progression, relapse, refractoriness, or T315I mutation). Subgroup analyses were also performed for patients who underwent an allogeneic stem cell transplantation before starting ponatinib treatment and for patients with MMR as best response to ponatinib. A sensitivity analysis was performed to investigate a potential imbalance in the CML patient population due to the inclusion of advanced phase CML patients.

\section{Results}

\section{Patient characteristics}

A total of 33 patients with CML and 17 with Ph+ ALL were included in this registry (Table 1). Among the CML patients, 30 were in chronic phase, 1 in accelerated phase, 1 in blastic myeloid phase, and 1 in blastic lymphoid phase. Out of 11 $\mathrm{CML}$ and $10 \mathrm{Ph}+\mathrm{ALL}$ patients with mutations in the $B C R$ $A B L 1$ kinase domain, $6(55 \%) \mathrm{CML}$ and $6(60 \%) \mathrm{Ph}+\mathrm{ALL}$ patients had the T315I mutation. No mutations were detected in $19(58 \%)$ patients with CML and $7(41 \%)$ patients with $\mathrm{Ph}+\mathrm{ALL}$, and mutation status was not determined for 3 (9\%) patients with CML. Median age was 58 (19-83) years for CML and 56 (28-80) years for Ph+ ALL patients (Table 1). In total, $39 \%$ of CML and $35 \%$ of Ph+ ALL patients had a history of cardiovascular disease, $33 \%$ of $\mathrm{CML}$ and $35 \%$ of $\mathrm{Ph}+\mathrm{ALL}$ patients presented with arterial hypertension, $15 \%$ of CML and $18 \%$ of Ph+ ALL patients had diabetes mellitus, $12 \%$ of CML and $6 \%$ of Ph+ ALL 
Table 1 Patient baseline characteristics

\begin{tabular}{|c|c|c|c|}
\hline & $\begin{array}{l}\text { All patients } \\
(N=50)\end{array}$ & $\begin{array}{l}\text { CML patients } \\
(N=33)\end{array}$ & $\begin{array}{l}\mathrm{Ph}+\text { ALL patients } \\
(N=17)\end{array}$ \\
\hline Age in years, median (range) & $58(19-83)$ & $58(19-83)$ & $56(28-80)$ \\
\hline Age in years, mean (SD) & $56.2(15.56)$ & $56.8(15.62)$ & $55.1(15.86)$ \\
\hline Female, $n(\%)$ & $19(38 \%)$ & $12(36 \%)$ & $7(41 \%)$ \\
\hline \multicolumn{4}{|l|}{ Previous TKI lines, $n(\%)$} \\
\hline $1 \mathrm{TKI}$ & $4(8 \%)$ & $3(9 \%)$ & $1(6 \%)$ \\
\hline 2 TKIs & $23(46 \%)$ & $12(36 \%)$ & $11(65 \%)$ \\
\hline$\geq 3$ TKIs & $23(46 \%)$ & $18(55 \%)$ & $5(29 \%)$ \\
\hline \multicolumn{4}{|l|}{ Presence of mutations, $n(\%)$} \\
\hline$T 315 I$ & $12(24 \%)$ & $6(18 \%)$ & $6(35 \%)$ \\
\hline Other & $10(20 \%)$ & $5(15 \%)$ & $5(29 \%)^{\mathrm{a}}$ \\
\hline Not determined & $3(6 \%)$ & $3(9 \%)$ & - \\
\hline \multicolumn{4}{|l|}{ Medical history, $n(\%)$} \\
\hline Liver disorder & $2(4 \%)$ & - & $2(12 \%)$ \\
\hline Pancreas disorder & $2(4 \%)$ & $2(6 \%)$ & - \\
\hline Reduced kidney function & $6(12 \%)$ & $2(6 \%)$ & $4(24 \%)$ \\
\hline Hypertension & $17(34 \%)$ & $11(33 \%)$ & $6(35 \%)$ \\
\hline Cardiovascular disease & $19(38 \%)$ & $13(39 \%)$ & $6(35 \%)$ \\
\hline Smoking & $13(26 \%)$ & $10(30 \%)$ & $3(18 \%)$ \\
\hline Diabetes & $8(16 \%)$ & $5(15 \%)$ & $3(18 \%)$ \\
\hline Hyperlipidemia & $5(10 \%)$ & $4(12 \%)$ & $1(6 \%)$ \\
\hline Hypercholesterolemia & $6(12 \%)$ & $2(6 \%)$ & $4(24 \%)$ \\
\hline Significant alcohol abuse & $4(8 \%)$ & $4(12 \%)$ & - \\
\hline Other & $36(72 \%)$ & $21(64 \%)$ & $15(88 \%)$ \\
\hline
\end{tabular}

$C M L$ chronic myeloid leukemia; $P h+A L L$ Philadelphia chromosome-positive acute lymphoblastic leukemia; $T K I$ tyrosine kinase inhibitor; $S D$ standard deviation; $N$ total number of patients; $n$ number of patients in respective category

${ }^{\mathrm{a}}$ In total, there were $10 \mathrm{Ph}+\mathrm{ALL}$ patients with mutations. One patient had a T315I mutation and another mutation patients had hyperlipidemia, and $30 \%$ of CML and $18 \%$ of $\mathrm{Ph}+\mathrm{ALL}$ patients were smokers. One patient with $\mathrm{Ph}+$ ALL had a history of heart failure.

\section{Previous treatment}

Among patients with CML, 3 (9\%) received 1 previous line of TKI treatment (all with T315I mutation), 12 $(36 \%)$ received 2 previous lines, and $18(55 \%)$ received 3 or more previous lines (Table 1). The corresponding numbers among $\mathrm{Ph}+\mathrm{ALL}$ patients were $1(6 \%), 11$ (65\%), and 5 (29\%), respectively. None of the Ph+ ALL patients had received more than 3 lines of different TKIs. Imatinib was the most commonly used first-line TKI, being used by $22(67 \%) \mathrm{CML}$ and $16(94 \%) \mathrm{Ph}+\mathrm{ALL}$ patients, while dasatinib was the most common second-line TKI, used in 19 (58\%) CML and $14(82 \%) \mathrm{Ph}+\mathrm{ALL}$ patients. Nilotinib was used as a third-line therapy in 7 (21\%) CML and 4 (24\%) Ph+ ALL patients, and bosutinib as a fourth-line therapy in $4(12 \%)$ CML patients. Two (6\%) patients with CML and $6(35 \%)$ patients with $\mathrm{Ph}+$ ALL had received an allogeneic stem cell transplantation prior to ponatinib treatment.

\section{Ponatinib treatment}

Treatment with ponatinib was started in 14 patients with CML due to intolerance to previous TKI (42\%), in 6 patients due to progression on previous TKI (18\%), in 9 patients due to relapse or refractoriness (absence of response, primary refractoriness, hematological or cytogenetic relapse) to previous TKI (27\%), and in 4 patients due to the T315I mutation (12\%). Among Ph+ ALL patients, reasons to start treatment with ponatinib were intolerance to previous TKI $(6,35 \%)$, disease progression $(2,12 \%)$, relapse or refractoriness (absence of response, primary refractoriness, hematological or cytogenetic relapse [5, 30\%]), or the T315I mutation $(4,24 \%)$ (Fig. 2). 
All patients $(\mathrm{N}=50)$ :

- intolerant $(\mathrm{N}=20)$

- non-intolerant $(\mathrm{N}=30)$

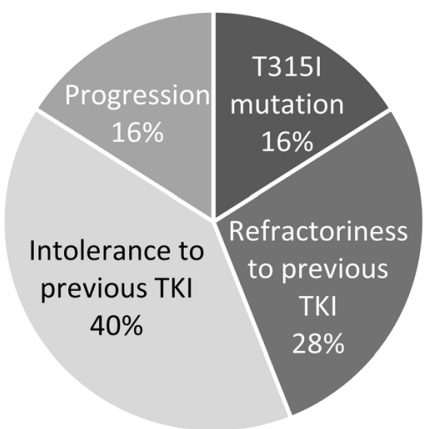

CML patients $(\mathrm{N}=33)$ :

- intolerant ( $N=14)$

- non-intolerant $(\mathrm{N}=19)$

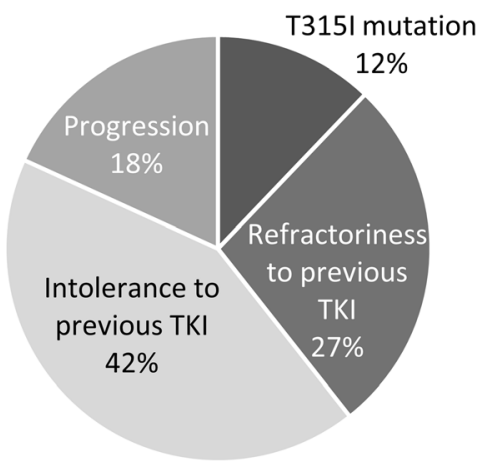

$\mathrm{Ph}+\mathrm{ALL}$ patients $(\mathrm{N}=17)$ :

- intolerant $(\mathrm{N}=6)$

- non-intolerant $(\mathrm{N}=11)$

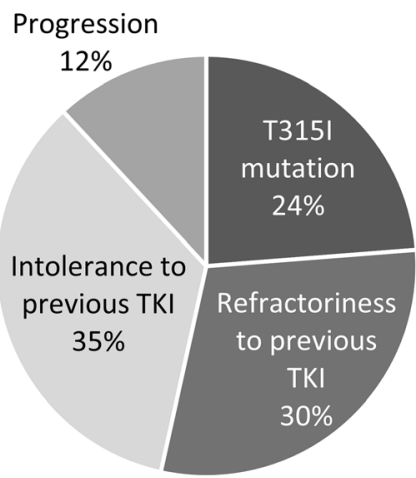

Fig. 2 Reasons for starting ponatinib treatment. CML, chronic myeloid leukemia; Ph+ ALL, Philadelphia chromosome-positive acute lymphoblastic leukemia; intolerant, those who started ponatinib due to intolerance to previous tyrosine kinase inhibitors (TKIs); non-intolerant, those who started ponatinib due to reasons other than intolerance to previous

The time from CML or Ph+ ALL diagnosis and the start of ponatinib treatment varied, ranging from 44 to 8139 days for CML patients and from 158 to 3632 days for $\mathrm{Ph}+\mathrm{ALL}$ patients. The majority of patients (70\% of CML and $76 \%$ of Ph+ ALL) received ponatinib at the starting dose of $45 \mathrm{mg} /$ day. The starting dose of $30 \mathrm{mg} /$ day was used in $12 \%$ of patients with CML and in $12 \%$ of patients with Ph+ ALL, and the starting dose of $15 \mathrm{mg} /$ day was used in $15 \%$ of patients with CML and in $12 \%$ of patients with $\mathrm{Ph}+\mathrm{ALL}$. One patient with CML started the therapy with $15 \mathrm{mg}$ of ponatinib every other day. There was a difference over time in the proportion of CML patients receiving the registered $45 \mathrm{mg}$ /day starting dose of ponatinib: Among the first 10 patients included in the registry, $86 \%$ of CML patients received a starting dose of $45 \mathrm{mg} /$ day, while in the last 10 included patients, only $43 \%$ of $\mathrm{CML}$ patients started with $45 \mathrm{mg} /$ day.

\section{Treatment outcomes}

The median follow-up was 449 days (range, 15-2777) and 135 days (range, 26-2114) for patients with $\mathrm{CML}$ and $\mathrm{Ph}+$ ALL, respectively. The median duration of ponatinib treatment was 380 days (range, 15-2777) for CML patients and 123 days (range, 13-2114) for Ph+ ALL patients. A swimmer plot detailing treatment duration and outcomes and of all individual patients in the registry is presented in Fig. 3.

MMR (BCR-ABL1 mRNA $\leq 0.1 \%)$ was achieved as best response by 26 patients: $19(58 \%)$ patients with CML and 7 (41\%) patients with $\mathrm{Ph}+$ ALL (Fig. 4). Of the CML patients who started with ponatinib at $45 \mathrm{mg} /$ day, $30 \mathrm{mg} /$ day, and 15 $\mathrm{mg} /$ day, respectively, 15 out of $23(65 \%)$, none out of 4 and 3 out of $5(60 \%)$ achieved MMR as best response. Also, the only CML patient starting at $15 \mathrm{mg}$ every other day achieved
TKIs (progression, T315I mutation, relapse or refractoriness). Refractoriness to previous TKI in this figure included absence of response, primary refractoriness, and hematological or cytogenetic relapse on previous TKI. Percentages may not add up to 100 due to rounding

MMR. Of the Ph+ ALL patients who started with $45 \mathrm{mg} /$ day, $30 \mathrm{mg} /$ day, and $15 \mathrm{mg} /$ day, respectively, 6 out of $13(46 \%)$, none out of 2 and 1 out of $2(50 \%)$ achieved MMR as best response. Two (6\%) patients with CML and $3(18 \%)$ patients with $\mathrm{Ph}+\mathrm{ALL}$ achieved CCyR as best response (including 1 patient with $\mathrm{Ph}+\mathrm{ALL}$ who had CCyR before starting ponatinib treatment) and 1 patient from each group (3\% and $6 \%$ of $\mathrm{CML}$ and $\mathrm{Ph}+\mathrm{ALL}$ patients, correspondingly) achieved $B C R-A B L 1$ mRNA $\leq 1 \%$ as best response. Nine (27\%) patients with CML and $2(12 \%)$ patients with $\mathrm{Ph}+$ ALL did not achieve any response. The median time to best response was 151 days (range, 26-616) for patients with CML and 49 days (range, 14-308) for patients with Ph+ ALL. Among the 20 patients who started ponatinib because of intolerance to previous TKIs, MMR was achieved as best response by $9(64 \%)$ CML and $4(67 \%) \mathrm{Ph}+$ ALL patients and 1 (7\%) CML patient achieved $B C R-A B L 1 \mathrm{mRNA} \leq 1 \%$ (Fig. 4). The median time to best response in patients who started ponatinib due to previous TKI intolerance was 159 days (range, 51-431) in patients with CML and 73 days (range, 14-308) in patients with $\mathrm{Ph}+\mathrm{ALL}$. Among the 30 patients who started ponatinib for reasons other than intolerance to previous TKI(s), 10 (53\%) patients with CML and 3 (27\%) patients with $\mathrm{Ph}+\mathrm{ALL}$ achieved MMR as best response. The median time to best response in these patients was 140 days (range, 26-616) and 36 days (range, 19-97), respectively. A sensitivity analysis, excluding the $3 \mathrm{CML}$ patients in accelerated or blastic phase, did not show any difference in the percentage of CML patients who started ponatinib due to intolerance and who achieved MMR as best response compared to those who did not show intolerance with MMR as best response.

Estimated OS was $85.3 \%$ for CML and $85.6 \%$ for $\mathrm{Ph}+$ ALL patients over 3 years of the registry duration. Estimated 


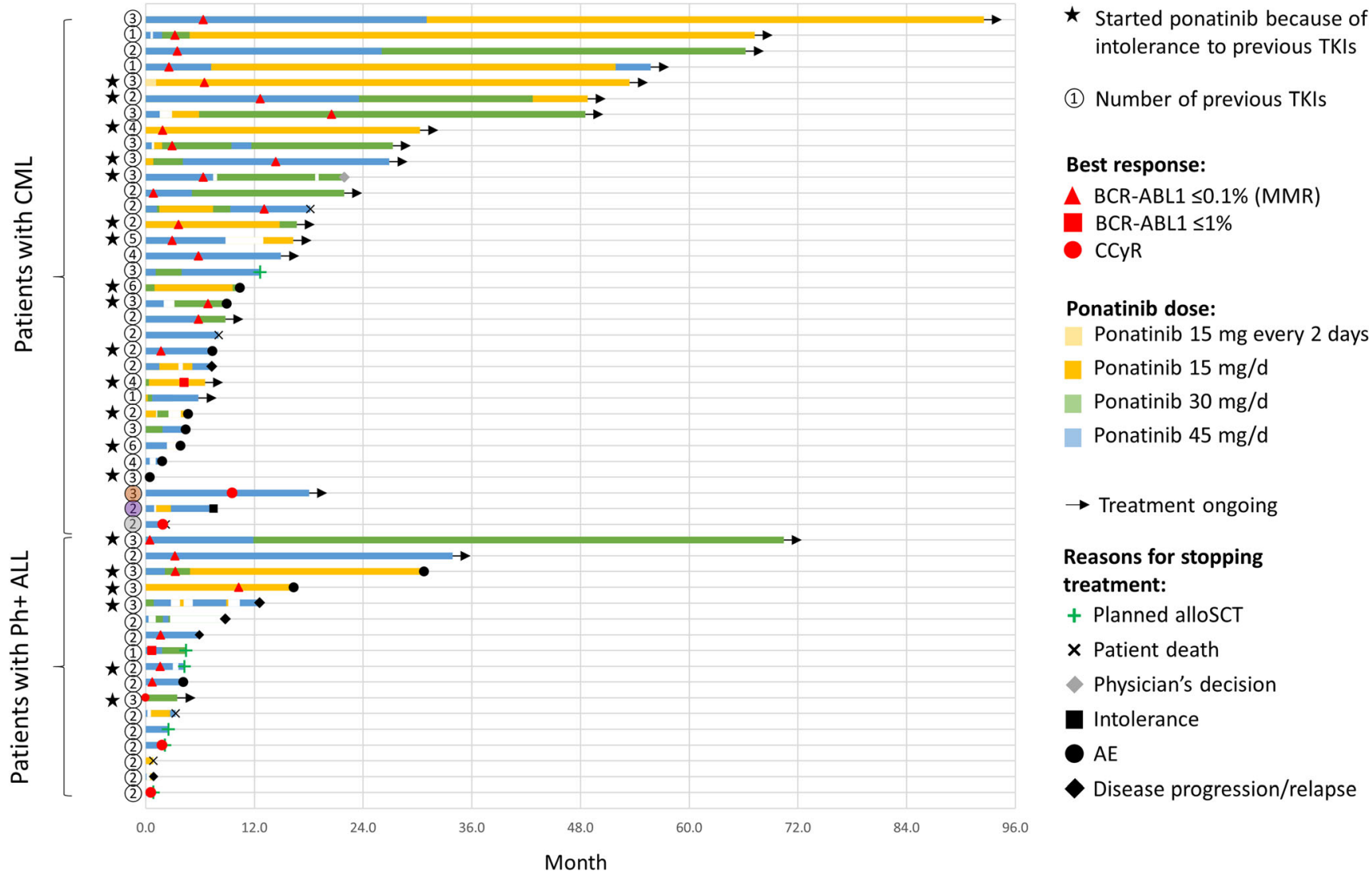

Fig. 3 Swimmer plot displaying treatment duration, treatment modification, and outcome for each patient. Each bar represents an individual patient. Patients with CML were in chronic phase, except for 3 indicated patients: orange circle, blastic myeloid phase; purple

PFS was $81.6 \%$ for CML and $48.9 \%$ for Ph+ ALL patients (Fig. 5).

Dose reductions occurred in $20(61 \%)$ CML and 7 (41\%) $\mathrm{Ph}+\mathrm{ALL}$ patients, and dose increases occurred in $14(42 \%)$ and $3(18 \%)$ patients with CML and $\mathrm{Ph}+\mathrm{ALL}$, respectively (Table 2). Dose reductions or interruptions occurred mostly due to AEs, but also to prevent future AEs as part of a risk management strategy in CML and Ph+ ALL patients. Reasons for dose increases in CML patients and Ph+ ALL patients were absent or poor response and good tolerance to a lower dose of ponatinib. Treatment interruptions were registered in $11(33 \%)$ CML and $5(29 \%) \mathrm{Ph}+$ ALL patients, with a median duration of interruption of 24 days (range, 7-126) in CML and 20 days (range, 12-201) in Ph+ ALL patients. In total, 15 CML $(45 \%)$ and $14(82 \%) \mathrm{Ph}+$ ALL patients terminated the treatment with ponatinib. Eight CML patients and $3 \mathrm{Ph}+\mathrm{ALL}$ patients terminated treatment due to an AE.

In general, results in the subgroup of patients who underwent an allogeneic stem cell transplantation before starting ponatinib were in line with the overall population. Of the 8 patients treated in the NPP who were included in this registry, 6 patients are currently still on treatment and circle, accelerated phase; grey circle, blastic lymphoid phase; AE, adverse event; CCyR, complete cytogenetic response; MMR, major molecular response; alloSCT, allogeneic stem cell transplantation; TKI, tyrosine kinase inhibitor

achieved MMR. These patients have been on ponatinib treatment from 4 up to 7 years.

\section{Safety}

Thirty-four (68\%) patients experienced adverse reactions. The most frequently reported adverse reactions (by $\geq 10 \%$ of patients) were rash and dry skin (Fig. 6). Other reported adverse reactions of interest included thrombocytopenia (4), abdominal pain (4), vascular stenosis (3), arterial hypertension (2), chest pain (1), palpitations (1), vascular occlusion (1), pancytopenia (1), increased serum lipase (1), cholecystitis (1), hepatitis (1), cholestasis (1), pneumonia (1), hyponatremia (1), pancreatitis (1), and hepatocellular injury (1).

In total, 5 deaths were registered; none of these was considered by the investigator to be a consequence of the ponatinib treatment. Three patients suffered from general physical health deterioration resulting in death and two patients died from cardiorespiratory arrest induced by euthanasia (one patient with relapse of $\mathrm{Ph}+\mathrm{ALL}$; one patient with both metastatic renal cell carcinoma and relapse of $\mathrm{Ph}+\mathrm{ALL}$ ). 
a

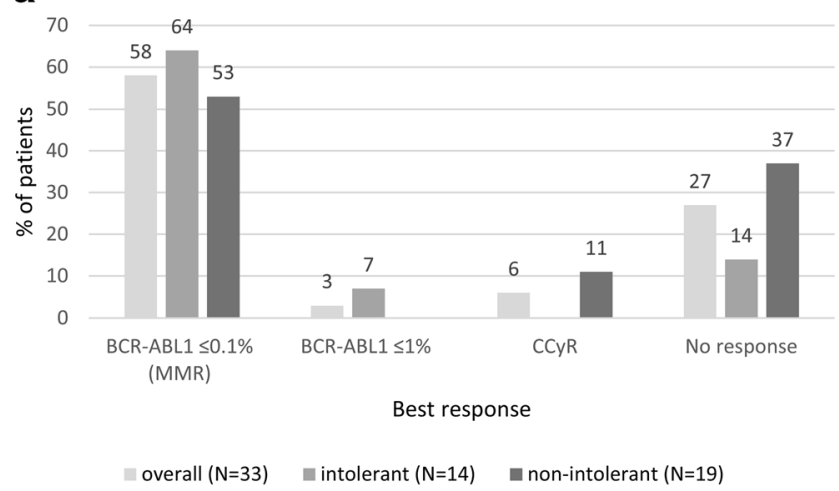

b

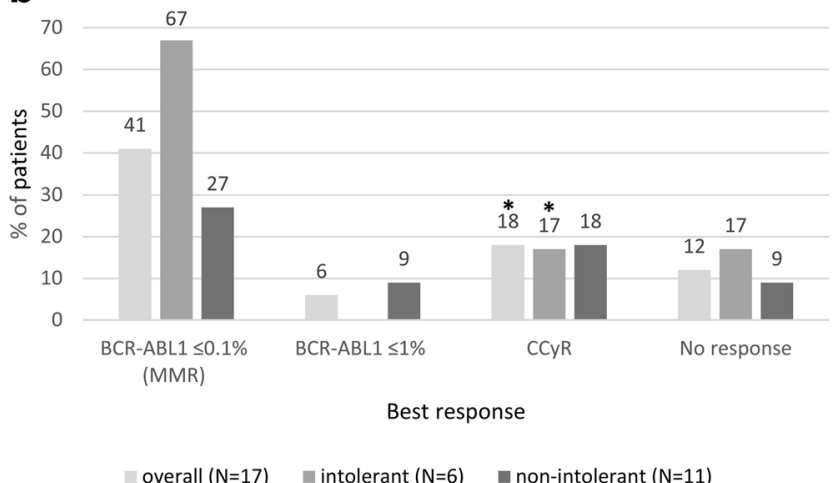

Fig. 4 Best response to treatment in patients with a CML and $\mathbf{b} \mathrm{Ph}+\mathrm{ALL}$ (overall, intolerant and non-intolerant patients). CCyR, complete cytogenetic response; CML, chronic myeloid leukemia; MMR, major molecular response; N, number of patients; Ph+ ALL, Philadelphia chromosomepositive acute lymphoblastic leukemia. *1 patient had achieved CCyR before starting treatment with ponatinib. Of note, $2(6 \%)$ patients with CML and $4(24 \%)$ patients with Ph+ ALL did not have measurable responses to treatment

\section{Discussion}

This registry collected data on the use of ponatinib in patients with $\mathrm{CML}$ or $\mathrm{Ph}+\mathrm{ALL}$ in routine clinical practice in Belgium. Data were collected in 20 hospitals that covered most of the patient population treated with ponatinib in the country. The results of this registry are in line with those of the PACE clinical trial [7, 8], although differences in patient population, size, study design, and follow-up hinder direct comparison. The median follow-up in this registry was 15 months for patients with CML and 4.5 months for patients with Ph+ ALL. A longer follow-up will be possible since this registry will continue for another 3 years. The number of patients harboring mutations in this registry was in line with PACE [8]. The overall percentage of patients with CML and Ph+ ALL who achieved MMR as best response was $58 \%$ and $41 \%$, respectively. It should be noted that information on patients' responses to any previous TKI (before starting the registry) was not collected. This limits the interpretation of the treatment responses as we do not know whether responses occurred rapidly after starting ponatinib or whether responses from previous treatment were maintained. For CP-CML patients, the duration of response in the registry was in line with the duration seen in the PACE trial. More than one-third of all patients (42\% CML and 35\% Ph+ ALL patients) started ponatinib treatment due to intolerance to previous TKIs.

Cross-intolerance to different TKIs is generally not considered to be clinically relevant, particularly for nonhematologic AEs [11]. While 20 patients (14 CML and 6 $\mathrm{Ph}+\mathrm{ALL}$ ) initially started ponatinib because of intolerance to previous TKIs, only 1 patient in this registry discontinued ponatinib treatment due to intolerance. This was a CML patient who had switched to ponatinib for another reason than intolerance. From the 20 patients who initially started ponatinib because of intolerance to previous TKIs, 11 discontinued ponatinib treatment, of which 8 due to an $\mathrm{AE}$ (73\%). From the 30 patients who started ponatinib for other reasons than intolerance, 18 discontinued ponatinib treatment, of which 3 due to an AE (17\%). Patients who discontinued due either to an $\mathrm{AE}$ or to treatment intolerance were considered to be intolerant in this analysis. It confirms that, even if some patients are intolerant to multiple TKIs, ponatinib can still be a valuable option for intolerant patients. While all patients in PACE initially started ponatinib at a dose of 45 $\mathrm{mg}$ /day, dose reductions were recommended subsequently in CP-CML patients who had achieved MCyR or MMR to reduce the risk for AOEs [8]. Most of those patients maintained their responses despite dose reductions. Based on the currently approved label, the starting dose of ponatinib is 45 $\mathrm{mg} /$ day. However, some patients in this registry received a lower starting dose. In the few patients who started with $15 \mathrm{mg} /$ day, this starting dose did not seem to preclude achieving MMR. A trend towards reduction of ponatinib starting dose in CML patients was observed during the course of this registry, as reflected by the proportion of patients receiving the maximal starting dose among the first and the last 10 patients enrolled in the registry. However, these results do not allow us to make any conclusions in this regard due to the small number of patients receiving each dose and current study design. While the recently updated recommendations from the European LeukemiaNet recommend a lower starting dose for certain CML patients [5], more data from randomized clinical trials are needed to further evaluate this. The effect of different starting doses of ponatinib (15 mg/day, $30 \mathrm{mg} /$ day, and $45 \mathrm{mg} /$ day) on the occurrence of AEs and on efficacy in CML patients is currently being evaluated in the prospective dose-ranging trial OPTIC (Optimizing Ponatinib Treatment in CML, clinicaltrials. gov identifier: NCT02467270).

Safety outcomes were similar to those observed in the PACE trial for the most frequently observed AEs. The incidence of cardiovascular AEs seemed low in this observational registry (reported for 8 patients [16\%]), even though patient 
a

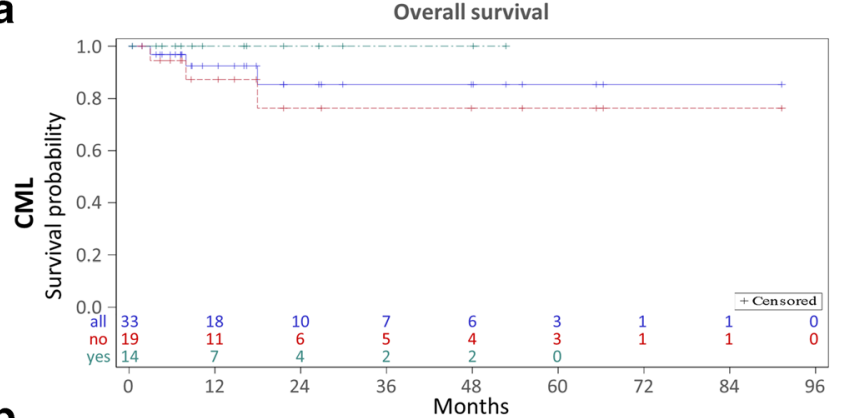

b

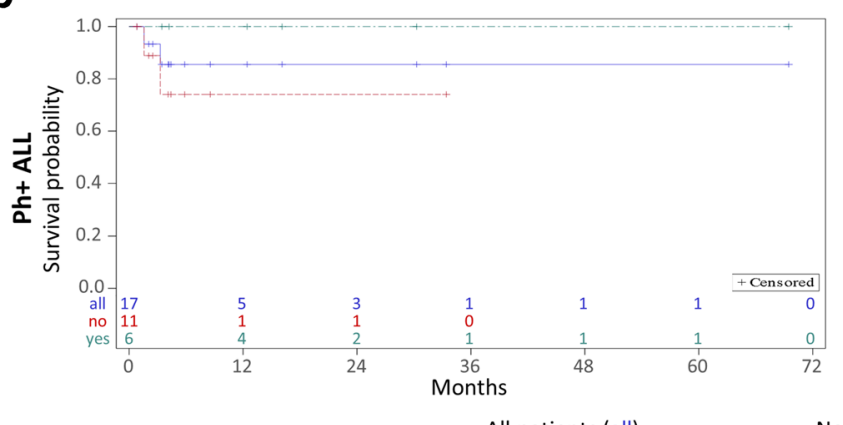

All patients (all)

Fig. 5 Kaplan-Meier estimates of overall survival and progression-free survival in a patients with CML (overall, intolerant and non-intolerant patients) and $\mathbf{b}$ patients with $\mathrm{Ph}+\mathrm{ALL}$ (overall, intolerant and nonintolerant patients). CML, chronic myeloid leukemia; Ph+ ALL,
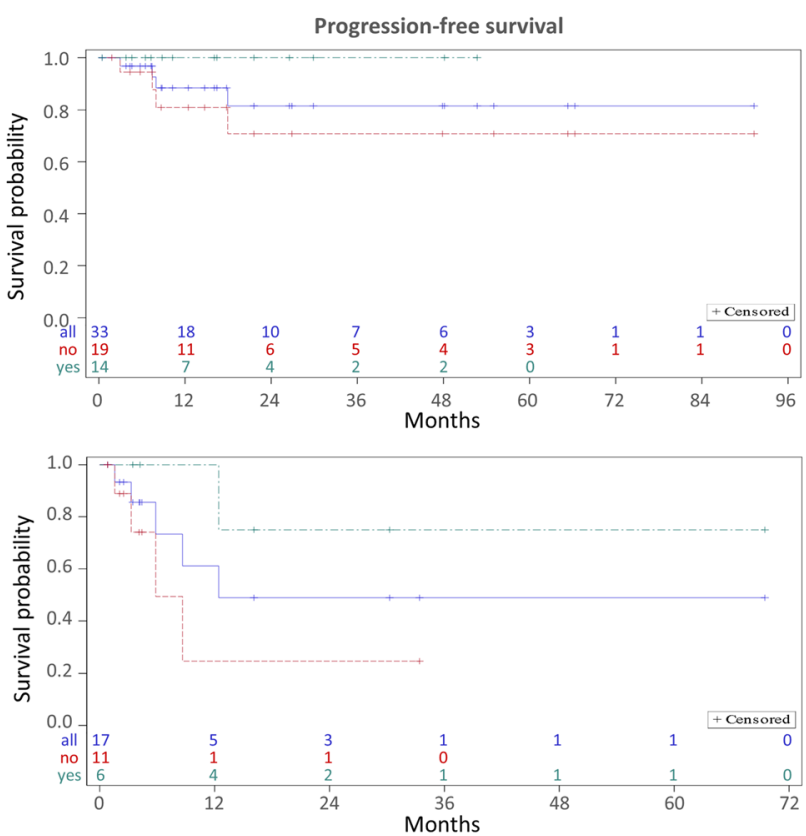

Philadelphia chromosome-positive acute lymphoblastic leukemia; TKI, tyrosine kinase inhibitor; OS, overall survival; PFS, progression-free survival
Table 2 Overview of and reasons for treatment modifications

\begin{tabular}{llll}
\hline & All patients & CML patients & Ph+ ALL patients \\
\hline Treatment modification, $n(\%)$ & $N=50$ & $N=33$ & $N^{\prime}=17$ \\
Dose reduction & $27(54)$ & $20(61)$ & $7(41)$ \\
Dose increase & $17(34)$ & $14(42)$ & $3(18)$ \\
Treatment interruption & $16(32)$ & $11(33)$ & $5(29)$ \\
Treatment termination & $29(58)$ & $15(45)$ & $14(82)$ \\
No change & $5(10)$ & $3(9)$ & $2(12)$ \\
Reasons for dose reduction/interruption, $n^{\prime}(\%)$ & $N^{\prime}=57$ & $N^{\prime}=38$ & $N^{\prime}=19$ \\
AE & $42(74)$ & $29(76)$ & $13(68)$ \\
Prevention & $14(25)$ & $9(24)$ & $5(26)$ \\
Other & $1(2)$ & - & $1(5)$ \\
Reasons for dose increase, $n^{\prime}(\%)$ & $N^{\prime}=24$ & $N^{\prime}=19$ & $N^{\prime}=5$ \\
No or low response & $11(46)$ & $10(53)$ & $1(20)$ \\
Good tolerance of treatment & $13(54)$ & $9(47)$ & $4(80)$ \\
Reasons for treatment termination, $n^{\prime}(\%)$ & $N^{\prime}=29$ & $N^{\prime}=15$ & $N^{\prime}=14$ \\
AE & $11(38)$ & $8(53)$ & $3(21)$ \\
Disease progression & $4(14)$ & $1(7)$ & $3(21)$ \\
Intolerance & $1(3)$ & $1(7)$ & - \\
Planned allogeneic stem cell transplantation & $6(21)$ & $1(7)$ & $5(36)$ \\
Other a & $7(27)$ & $4(27)$ & $3(21)$ \\
\hline
\end{tabular}

$A E$ adverse event; $N$ total number of patients; $N$ ' total number of treatment modifications (dose increase, dose decrease/interruption or treatment termination); $n$ (\%) number (percentage) of patients in respective category; $n$ ' (\%) number (percentage) of treatment modifications in respective category

${ }^{\text {a }}$ This includes the 5 deaths in the registry 
Fig. 6 The most frequently reported adverse reactions. $\mathrm{N}$, number of patients

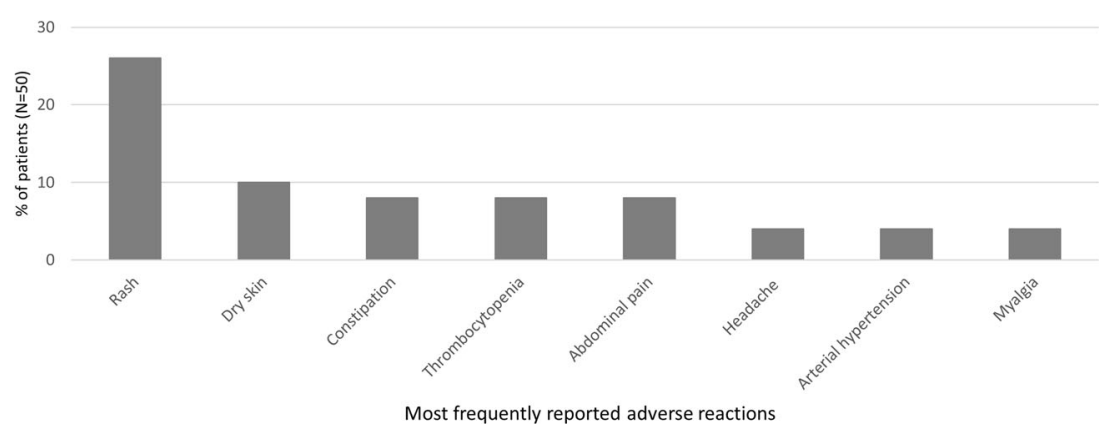

baseline characteristics might indicate certain risk factors for cardiovascular events. In the PACE trial, $31 \%$ of CP-CML and $25 \%$ of $\mathrm{Ph}+\mathrm{ALL}$ patients reported treatment-emergent AOEs of any grade. This difference could be due to the appropriate patient selection and monitoring, the implementation of the risk minimization activities, and a possible underreporting of AEs in a real-life situation as compared to a clinical trial or shorter follow-up period; this difference was also observed for the frequency of other AEs. The Common Terminology Criteria for Adverse Events grading was not collected for the purpose of this registry, limiting the clinical interpretation (severity) of the described AEs.

Several retrospective observational studies reporting clinical use of ponatinib have been published [12-14]. However, to the best of our knowledge, this Belgian registry is the first to prospectively evaluate the effectiveness and safety of ponatinib in routine clinical practice. This nationwide registry collected quality data from leading hospitals that treat $\mathrm{CML}$ and $\mathrm{Ph}+$ ALL patients in Belgium. Although the number of patients included in the registry was slightly higher compared to published retrospective studies, the available number of patients per diagnosis was still too small to allow robust statistical analysis. Hence, all analyses in this registry were descriptive.

In conclusion, the results obtained in routine clinical practice in Belgium are in line with those of the PACE trial, therefore supporting the use of ponatinib in patients with $\mathrm{CML}$ and $\mathrm{Ph}+\mathrm{ALL}$ after failure or intolerance to previous lines of TKIs or who have the T315I mutation. Major molecular responses were observed as best response in most CML patients and a large proportion of Ph+ ALL patients. In $\mathrm{Ph}+\mathrm{ALL}$ patients, deep molecular responses seemed to occur more frequently in intolerant versus non-intolerant patients. The registry revealed no new safety signals other than those previously reported $[7,8]$. The collection of registry data is still ongoing, with the aim of evaluating more patients and allowing for longer follow-up.

Supplementary Information The online version contains supplementary material available at https://doi.org/10.1007/s00277-021-04507-x.
Acknowledgements The authors would like to thank the patients, their caregivers, the clinical investigators, and research personnel for their contribution to this registry. The authors also would like to thank Ekaterina Ivanova and Kristel Vercauteren (Modis, Wavre, Belgium) for providing medical writing and publication coordination support.

Author contribution TD, VH, KT, SM, FSB, AG, GV, HV, BB, NG, PL, $\mathrm{ADB}, \mathrm{KVE}, \mathrm{MJ}, \mathrm{ML}, \mathrm{AT}, \mathrm{IV}, \mathrm{DD}$, and DM participated to the study as principal investigators. $\mathrm{MB}$ contributed to the interpretation of the results. All authors discussed the results, provided feedback, and contributed to the final manuscript.

Funding This study, medical writing, and publication coordination were supported by research funding from Incyte Biosciences Benelux BV.

Data availability The datasets generated during and/or analyzed during the current study are available from the corresponding author on reasonable request.

\section{Declarations}

Informed consent Informed consent was obtained from all individual participants included in the registry, but a waiver was granted by the ethics committee for patients in the Named Patient Program who were still on ponatinib treatment on March 1, 2016 but deceased before the registry start.

Research involving human participants and/or animals This registry was conducted in accordance with the ethical standards of the institutional and/or national ethics committees and with the Declaration of Helsinki and the International Council for Harmonization guidelines for good clinical practice.

Conflict of interest KT reports membership of advisory boards from multiple pharmaceutical companies. BB reports research funding from Incyte Biosciences Benelux BV. ADB reports ad hoc membership of advisory boards from multiple pharmaceutical companies. DD reports consultancy, honoraria, membership of Board of Directors or advisory committees, and research funding from multiple pharmaceutical companies. DS reports consultancy and travel expenses from Incyte Biosciences Benelux BV. JB and MB are employees of Incyte Biosciences. There are no relationships to disclose for TD, VH, SM, FSB, AG, GV, HV, NG, PL, KVE, MJ, AT, IV, and DM.

Open Access This article is licensed under a Creative Commons Attribution 4.0 International License, which permits use, sharing, adaptation, distribution and reproduction in any medium or format, as long as you give appropriate credit to the original author(s) and the source, provide a link to the Creative Commons licence, and indicate if changes were 
made. The images or other third party material in this article are included in the article's Creative Commons licence, unless indicated otherwise in a credit line to the material. If material is not included in the article's Creative Commons licence and your intended use is not permitted by statutory regulation or exceeds the permitted use, you will need to obtain permission directly from the copyright holder. To view a copy of this licence, visit http://creativecommons.org/licenses/by/4.0/.

\section{References}

1. Huang X, Cortes J, Kantariian H (2012) Estimations of the increasing prevalence and plateau prevalence of chronic myeloid leukemia in the era of tyrosine kinase inhibitor therapy. Cancer 118(12): 3123-3127. https://doi.org/10.1002/cncr.26679

2. Druker BJ (2008) Translation of the Philadelphia chromosome into therapy for CML. Blood 112(13):4808-4817. https://doi.org/10. 1182/blood-2008-07-077958

3. Garcia-Gutierrez V, Hernandez-Boluda JC (2019) Tyrosine kinase inhibitors available for chronic myeloid leukemia: efficacy and safety. Front Oncol 9:603. https://doi.org/10.3389/fonc.2019. 00603

4. Chopade P, Akard LP (2018) Improving outcomes in chronic myeloid leukemia over time in the era of tyrosine kinase inhibitors. Clin Lymphoma Myeloma Leuk 18(11):710-723. https://doi.org/ 10.1016/j.clml.2018.06.029

5. Hochhaus A, Baccarani M, Silver RT, Schiffer C, Apperley JF, Cervantes F, Clark RE, Cortes JE, Deininger MW, Guilhot F, Hjorth-Hansen H, Hughes TP, Janssen J, Kantarjian HM, Kim DW, Larson RA, Lipton JH, Mahon FX, Mayer J, Nicolini F, Niederwieser D, Pane F, Radich JP, Rea D, Richter J, Rosti G, Rousselot P, Saglio G, Saußele S, Soverini S, Steegmann JL, Turkina A, Zaritskey A, Hehlmann R (2020) European LeukemiaNet 2020 recommendations for treating chronic myeloid leukemia. Leukemia 34(4):966-984. https://doi.org/10.1038/ s41375-020-0776-2

6. O'Hare T, Shakespeare WC, Zhu X, Eide CA, Rivera VM, Wang F, Adrian LT, Zhou T, Huang WS, Xu Q, Metcalf CA 3rd, Tyner JW, Loriaux MM, Corbin AS, Wardwell S, Ning Y, Keats JA, Wang Y, Sundaramoorthi R, Thomas M, Zhou D, Snodgrass J, Commodore L, Sawyer TK, Dalgarno DC, Deininger MW, Druker BJ, Clackson T (2009) AP24534, a pan-BCR-ABL inhibitor for chronic myeloid leukemia, potently inhibits the T315I mutant and overcomes mutation-based resistance. Cancer Cell 16(5):401-412. https://doi. org/10.1016/j.ccr.2009.09.028

7. Cortes JE, Kim DW, Pinilla-Ibarz J, le Coutre P, Paquette R, Chuah C, Nicolini FE, Apperley JF, Khoury HJ, Talpaz M, DiPersio J, DeAngelo DJ, Abruzzese E, Rea D, Baccarani M, Muller MC, Gambacorti-Passerini C, Wong S, Lustgarten S, Rivera VM, Clackson T, Turner CD, Haluska FG, Guilhot F, Deininger MW, Hochhaus A, Hughes T, Goldman JM, Shah NP, Kantarjian H, Investigators P (2013) A phase 2 trial of ponatinib in Philadelphia chromosome-positive leukemias. N Engl J Med 369(19):17831796. https://doi.org/10.1056/NEJMoa1306494

8. Cortes JE, Kim DW, Pinilla-Ibarz J, le Coutre PD, Paquette R, Chuah C, Nicolini FE, Apperley JF, Khoury HJ, Talpaz M, DeAngelo DJ, Abruzzese E, Rea D, Baccarani M, Muller MC, Gambacorti-Passerini C, Lustgarten S, Rivera VM, Haluska FG, Guilhot F, Deininger MW, Hochhaus A, Hughes TP, Shah NP, Kantarjian HM (2018) Ponatinib efficacy and safety in Philadelphia chromosome-positive leukemia: final 5-year results of the phase 2 PACE trial. Blood 132(4):393-404. https://doi.org/ 10.1182/blood-2016-09-739086

9. Iclusig (ponatinib) Summary of Product Characteristics (2018). https://www.ema.europa.eu/en/documents/product-information/ iclusig-epar-product-356information_en.pdf. Accessed March 2021

10. Baccarani M, Deininger MW, Rosti G, Hochhaus A, Soverini S, Apperley JF, Cervantes F, Clark RE, Cortes JE, Guilhot F, HjorthHansen H, Hughes TP, Kantarjian HM, Kim DW, Larson RA, Lipton JH, Mahon FX, Martinelli G, Mayer J, Muller MC, Niederwieser D, Pane F, Radich JP, Rousselot P, Saglio G, Saussele S, Schiffer C, Silver R, Simonsson B, Steegmann JL, Goldman JM, Hehlmann R (2013) European LeukemiaNet recommendations for the management of chronic myeloid leukemia: 2013. Blood 122(6):872-884. https://doi.org/10.1182/blood-201305-501569

11. Muller MC, Cervantes F, Hjorth-Hansen H, Janssen J, Milojkovic D, Rea D, Rosti G (2017) Ponatinib in chronic myeloid leukemia (CML): consensus on patient treatment and management from a European expert panel. Crit Rev Oncol Hematol 120:52-59. https://doi.org/10.1016/j.critrevonc.2017.10.002

12. Breccia M, Abruzzese E, Castagnetti F, Bonifacio M, Gangemi D, Sora F, Iurlo A, Luciano L, Gozzini A, Gentile M, Bocchia M, Luzi D, Maggi A, Sgherza N, Isidori A, Crugnola M, Pregno P, Scortechini AR, Capodanno I, Pizzuti M, Foa R (2018) Ponatinib as second-line treatment in chronic phase chronic myeloid leukemia patients in real-life practice. Ann Hematol 97(9):1577-1580. https://doi.org/10.1007/s00277-018-3337-2

13. Heiblig M, Rea D, Chretien ML, Charbonnier A, Rousselot P, Coiteux V, Escoffre-Barbe M, Dubruille V, Huguet F, Cayssials E, Hermet E, Guerci-Bresler A, Ame S, Sackmann-Sala L, Roy L, Sobh M, Morisset S, Etienne G, Nicolini FE (2018) Ponatinib evaluation and safety in real-life chronic myelogenous leukemia patients failing more than two tyrosine kinase inhibitors: the PEARL observational study. Exp Hematol 67:41-48. https://doi. org/10.1016/j.exphem.2018.08.006

14. Shacham-Abulafia A, Raanani P, Lavie D, Volchek Y, Ram R, Helman I, Shargian L, Gourevitch A, Chubar E, Ratzon R, Rozovski U (2018) Real-life experience with ponatinib in chronic myeloid leukemia: a multicenter observational study. Clin Lymphoma Myeloma Leuk 18(7):e295-e301. https://doi.org/10. 1016/j.clml.2018.05.002

Publisher's note Springer Nature remains neutral with regard to jurisdictional claims in published maps and institutional affiliations. 